\title{
Children primed and ready for SARS-CoV-2
}

\author{
Single-cell sequencing of nasal swab samples from people uninfected or infected with SARS-CoV-2 shows that \\ children have a primed innate immune response, which may protect them from severe disease.
}

\section{Emily Speranza}

oronavirus disease 2019 (COVID-19),
a disease caused by infection with
severe acute respiratory syndrome
coronavirus-2 (SARS-CoV-2), can have
devastating outcomes, particularly in the
elderly. Advanced age is an important risk
factor for severe disease and death, whereas
young children often develop only mild
disease or may even have asymptomatic
infections. How children control infection
is incompletely understood. To address this
question, Loske et al. ${ }^{1}$ carried out single-cell
RNA sequencing (scRNA-seq) of nasal swab
samples from healthy children and adults,
as well as from children and adults with

mild-to-moderate COVID-19, and report their findings in Nature Biotechnology. They found that uninfected children have more immune cells in their nasal passage and that those cells, along with the epithelial cells, have a 'pre-activated' signature that is not present in uninfected adults. Children also had stronger innate immune responses than adults after infection with SARS-CoV-2, which suggests that pre-activation may help to control SARS-CoV-2 infection in children.

The type I interferon pathway helps to protect cells from viruses and recruits immune cells to the site of infection, thus playing an important role in controlling viral infection. COVID-19 severity has been linked to a dysregulation of the type I and type III interferon signalling pathways ${ }^{2}$. Some patients who present with severe disease even have autoantibodies against type I interferon, suggesting that the ability to turn on the type I interferon response is important in controlling virus-associated damage $^{3}$. Additionally, several SARS-CoV-2 proteins can block or dampen this response ${ }^{4}$, highlighting the importance of this pathway in determining the outcome of COVID-19. Innate immune cells are key producers of type I interferon, and they themselves, as

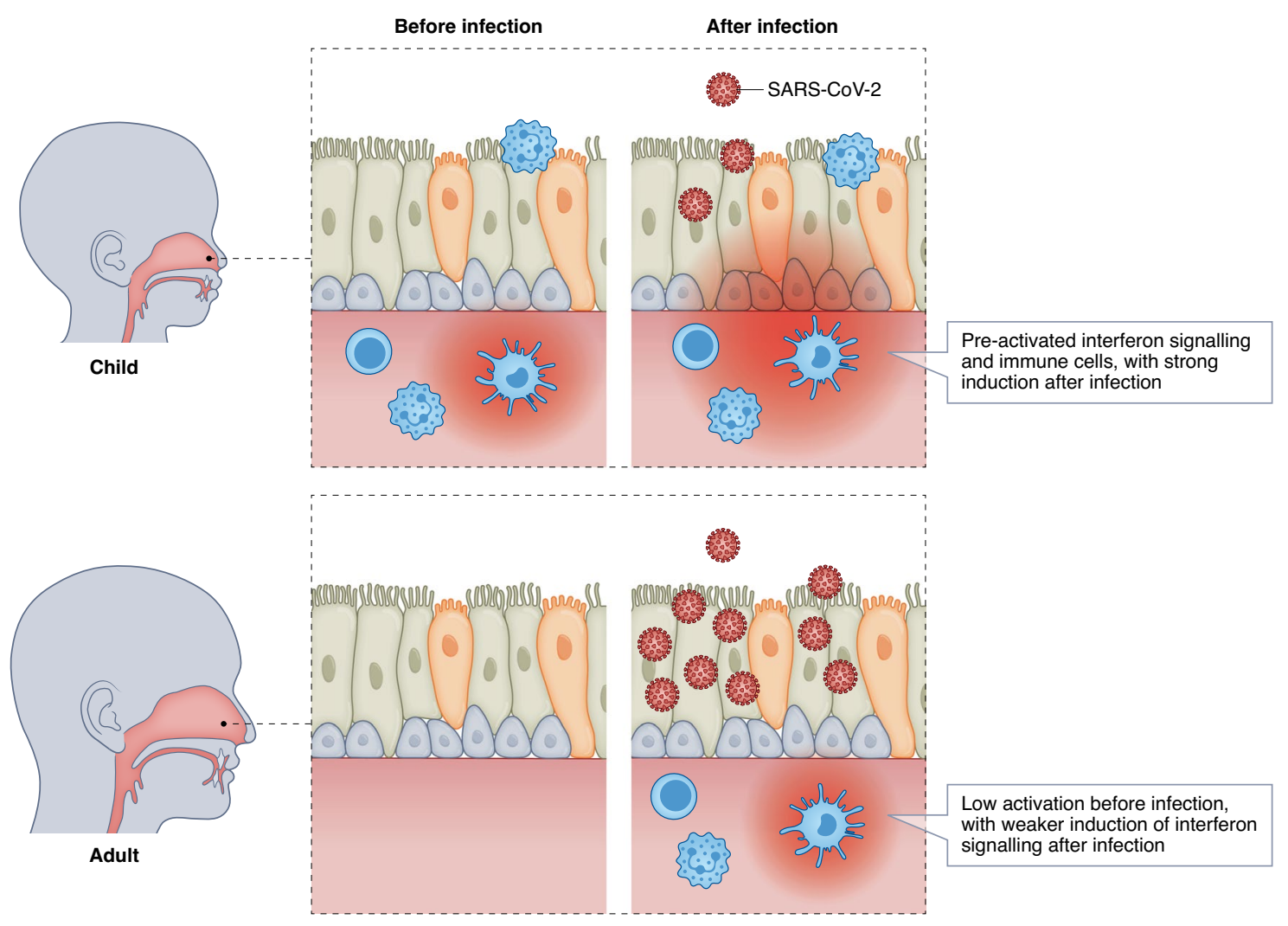

Fig. 1 | SARS-CoV-2 host response in children and adults profiled by scRNA-seq. Uninfected children showed higher levels of innate signalling than adults in epithelial and immune cells. Additionally, they had higher levels of neutrophils and certain types of cytotoxic T cells. After infection, both children and adults saw an upregulation of innate signalling in epithelial and immune cells, which was stronger in children. Cell numbers stayed relatively unchanged in children, whereas infection in adults let to an influx of immune cells. 
well as nasal epithelial cells, respond by upregulating interferon-stimulated genes (ISGs). When assessing the frequencies of cells in the nasal swab samples, Loske et al. found more immune cells, particularly neutrophils and cytotoxic T cells, in samples from children than in those from adults. Additionally, nasal epithelial cells, which are susceptible to SARS-CoV-2 infection, showed an up-regulation of the pattern-recognition receptor MDA5 and other ISGs in children, even before exposure to the virus. Having a primed immune response means that, following infection, children can increase the expression of important ISGs in epithelial cells, including ciliated cells and goblet cells, which potentially protects them from additional infection and helps to stop virus dissemination. (Fig. 1)

Epithelial cells were not the only cell type with a primed response that was heightened in children both before and after infection. Neutrophils, which were present in high numbers in children before infection, displayed rapid induction of activation-associated genes. Myeloid-derived cells, such as macrophages and dendritic cells, showed higher cell-to-cell interactions and interferon responsiveness before and during infection in children as compared to adults, suggesting an important role for innate immune cells in counteracting the virus. Finally, a subset of cytotoxic T cells that helps to sustain virus-specific responses was present in children before infection and was absent in adults. This difference may explain why children also had a higher expression of genes related to a cytotoxic T-cell response, such as Ifng (encoding interferon- $\gamma$ ), during infection.
One important aspect of infection not covered in this study is how the response in children compares with that observed in more severe disease in adults. Although it is important to isolate the features of protection observed in clinically matched samples to highlight the changes unique to age, an additional comparison to patients with a wider range of disease outcomes could help to contextualize which features of the young immune response correlate with the prevention of severe COVID-19 symptoms. Additionally, it will be important to understand the differences in asymptomatic infections, which the authors were unable to address due to the low number of asymptomatic patients. Despite these limitations, this work is still an important resource that highlights the differences associated with age that are observed during SARS-CoV-2 infection.

These findings shine a light on the role of the host innate immune response in controlling SARS-CoV-2. The innate immune response has an important role in controlling a viral infection in the absence of pre-existing immunity, especially in older populations. These data are especially timely as they coincide with the spread of the Delta variant, also called B.1.617.2, and the return to school for many unvaccinated children. As children continue to test positive for SARS-CoV-2, understanding the response in the airway before infection and how this changes during a mild-to-moderate disease course provides a baseline for assessing a protective response in children and may inform ways of stimulating protective mucosal immunity in children and adults.

The report by Loske et. al., together with other studies ${ }^{5,6}$, highlights how scRNA-seq studies have enhanced our understanding of the pathogenesis of COVID-19. scRNA-seq has been applied to profile cells susceptible to SARS-CoV-2 infection in cohorts of varying age and sex and with different comorbidities $^{7}$ and host immune response dynamics in the upper and lower airways ${ }^{8}$.

The ability of scRNA-seq both to identify host signalling pathways that are important in viral control or the recovery from disease and to link those pathways to the specific cells that produce the signal will inform the design of vaccines and host-based therapeutics.

\section{Emily Speranza (D)}

Lymphocyte Biology Section, Laboratory of Immune System Biology, National Institute of Allergy and Infectious Disease, National Institutes of Health, Bethesda, MD, USA.

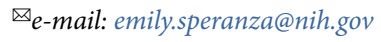

Published online: 20 October 2021 https://doi.org/10.1038/s41564-021-00984-y

References

1. Loske, J. et al. Nat. Biotechnol. https://doi.org/10.1038/s41587021-01037-9 (2021)

2. Choi, H. \& Shin, E. C. Yonsei Med. J. 62, 381-390 (2021).

3. Bastard, P. et al. Science https://doi.org/10.1126/science.abd 4585 (2020).

4. Lei, X. et al. Nat. Commun. 11, 3810 (2020).

5. Chua, R. L. et al. Nat. Biotechnol. 38, 970-979 (2020).

6. Trump, S. et al. Nat. Biotechnol. 39, 705-716 (2021).

7. Muus, C. et al. Nat. Med. 27, 546-559 (2021).

8. Liao, M. et al. Nat. Med. 26, 842-844 (2020).

\section{Acknowledgements}

The author is supported by the intramural research program of the NIH.

Competing interests

The author declares no competing interests. 